\title{
Nanometre-scale electrochemical switches fabricated using a plasma-based sulphidation technique.
}

\author{
Andrew F. Thomson, David O. S. Melville and Richard J Blaikie* \\ MacDiarmid Institute for Advanced Materials and Nanotechnology \\ Department of Electrical and Computer Engineering \\ University of Canterbury, Christchurch 8020, New Zealand \\ Email: r.blaikie@elec.canterbury.ac.nz \\ Telephone: +64 (3) 364 7001, Fax: +64 (3) 3642761
}

\begin{abstract}
Solid-state electrochemical switches are new devices that may find application in low power logic or memory circuits. The reversible electrochemical oxidation and reduction of a sub 10nm-thick silver sulphide layer is the critical process for these devices' operation, which determines the switching speed and the OFF/ON resistance ratio. A new process has been developed for fabricating QCAS devices, including a novel $\mathrm{SF}_{6}$ plasma sulphidation technique for forming the critical silver sulphide switchable insulator layer. QCAS devices were successfully created with $O F F / O N$ resistance ratios of up to $10^{6}$, with switching speeds comparable to previous devices and far less problematic yields.
\end{abstract}

Keywords-electrical switching; solid-state electrolytes; nanoelectronics; non-volatile memory

\section{INTRODUCTION}

The Quantised Conductance Atomic Switch (QCAS) [1] belongs to a class of solid-state electrochemical switching devices $[2,3,4]$ that offer simple and robust operation, scalable to lateral dimensions below $50 \mathrm{~nm}$. These utilise reversible electrochemical reduction and oxidation of metal sulphides (silver or copper) to control nano-protrusions (Fig. 1), thereby acting as two-terminal memory devices. The formation of high-quality sub-10 $\mathrm{nm}$ sulphide layers is critical for device operation, and determines the switching speed, reliability and the $\mathrm{OFF} / \mathrm{ON}$ resistance ratio.

In this paper we discuss work that we have been undertaking in this area, in which a series of QCAS devices were not only successfully recreated, but with a new and innovative plasma-based sulphidation technique. The ON/OFF ratio of our devices is superior to those in Ref. [1], however the switching characteristics are not as good.

\section{THEORY OF OPERATION}

A QCAS is a simple two terminal device consisting of two electrodes separated by silver sulphide $\left(\mathrm{Ag}_{2} \mathrm{~S}\right)$ acting as a dielectric insulator as shown in Fig. 1(a). When a high enough potential is applied across the terminals a reversible dielectric breakdown occurs creating a low resistance path; the device's state can be measured at lower potentials without affecting its state. The changing conductance of this device is due to the formation and disappearance of silver ( $\mathrm{Ag}$ ) nano-protrusions, via a reversible redox and diffusion reaction to make the connection between $\mathrm{Ag}_{2} \mathrm{~S}$ coated $\mathrm{Ag}$ electrode and a platinum (Pt) electrode. K. Terabe et al [2] describe the phenomena of formation and disappearance of these protrusions, although it is not yet fully understood.

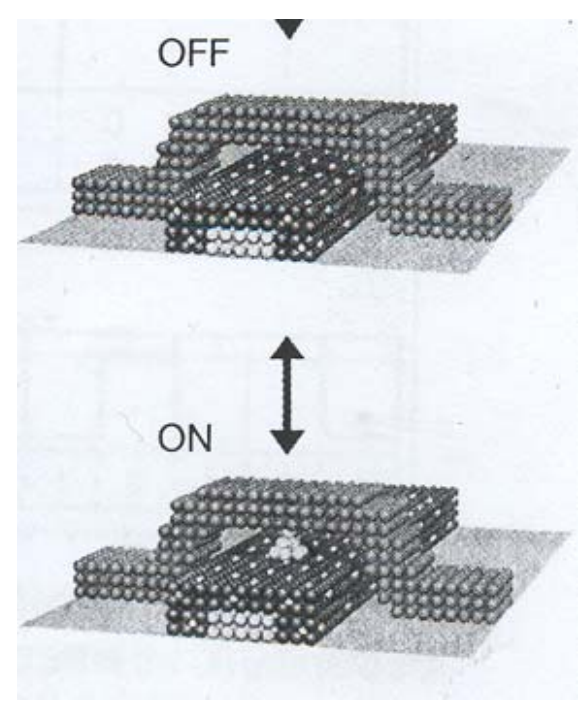

Figure 1. Schematic diagram of the QCAS device operation (from ref [1]) Nano-protrusions are reversibly formed and broken between a sulphide-coated bottom electrode and an inert-metal control electrode above.

\section{A. Original QCAS Results}

The original QCAS devices were quoted as having $1 \mathrm{MHz}$ switching speed with a $600 \mathrm{mV}$ operational voltage at room temperature [1]. The operational resistances of the devices were quoted to be $2 \mathrm{k} \Omega$ in the ON state and $100 \mathrm{k} \Omega$ in the OFF state; giving an $\mathrm{OFF} / \mathrm{ON}$ resistance ratio of approximately 50 . It was stated that they believed $1 \mathrm{GHz}$ switching is possible once the device capacitance is reduced. At present levels of miniaturisation the storage capacity of the devices was quoted 
as being in the order of $2.5 \mathrm{Gbits} / \mathrm{cm}^{2}$. Yields for these devices were not explicitly stated, only said to be problematic.

\section{B. Sulphidation Techniques}

Previous reports of solid-state electrochemical switching devices have used liquid-phase [2] or exotic vapour-phase methods [1] for forming the metal sulphide layer. For example, an ultra-high vacuum (UHV) sulphur vapour reaction at $80^{\circ} \mathrm{C}$ for 5 minutes was used to form the critical $\mathrm{Ag}_{2} \mathrm{~S}$ coated $\mathrm{Ag}$ wires in Ref[1]. This is a non-conventional semiconductor processing step that is not widely available in research laboratories, so alternative sulphidation techniques were investigated.

The work of Korvas et al. [5] considered the growth of $\mathrm{Ag}_{2} \mathrm{~S}$ films in air of over a long period of time. A similar but faster method was considered by Mehan and Mansingh [6], where the silver was sulphidised in a $50 \% \mathrm{H}_{2} \mathrm{~S}$ atmosphere. These techniques were either too slow or too dangerous for us to use, and also did not involve the use of standard microelectronics processing equipment. Hence a new sulphidation process technique was developed, using energetic sulphur ions derived from a $\mathrm{SF}_{6}$ plasma in a reactive ion etching (RIE) tool (Oxford Instruments PlasmaLab80). By using a low-power etch (Table 1) surface damage can be minimised and smooth, uniform sulphide layers can be formed.

TABLE I. SilVER SUlPHIDATION Process PARAMETERS

\begin{tabular}{|c|c|c|c|c|c|}
\hline Gas & $\begin{array}{c}\text { Flow Rate } \\
\text { (sccm) }\end{array}$ & $\begin{array}{c}\text { Pressure } \\
\text { (Torr) }\end{array}$ & $\begin{array}{c}\text { RF Power } \\
\text { (W) }\end{array}$ & $\begin{array}{c}\text { Temp } \\
\text { (K) }\end{array}$ & $\begin{array}{c}\text { Time } \\
\text { (s) }\end{array}$ \\
\hline $\mathrm{SF}_{6}$ & 80 & 0.25 & 40 & 300 & 90 \\
\hline
\end{tabular}

\section{DEVICE FABRICATION}

Device fabrication consists of four main tasks: creation of contact pads, creation of $\mathrm{Ag}$ wires, sulphidation of $\mathrm{Ag}$ wires, and creation of Pt crossbars. The goal of each of these fabrication tasks was to create a device as similar to the specifications of Ref [1] as possible, so that differences due to the new $\mathrm{SF}_{6}$-based sulphidation could be assessed.

\section{A. Device Specifications}

The SEM image in Fig. 2 shows the electrode layout for the device reported in Ref. [1]. The vertical wire is a sulphidised $\mathrm{Ag}$ wire approximately $150 \mathrm{~nm}$ wide; this is overlaid by two horizontal Pt crossbars approximately $100 \mathrm{~nm}$ wide. It was claimed that there is a $1 \mathrm{~nm}$ gap between the two electrodes, formed by the deposition of a 1-nm thick Ag layer, which was later removed by the charging and discharging of the $\mathrm{Pt}$ electrode with a positive bias. However we have not tried to reproduce this step, and our devices still work well.

It is important to note that this device is a two terminal device; the second Pt crossbar in Fig. 2 is not necessary, nor is it necessary to contact each wire twice. This has been done to allow two devices to be created closer together, and to help in the testing of the devices.

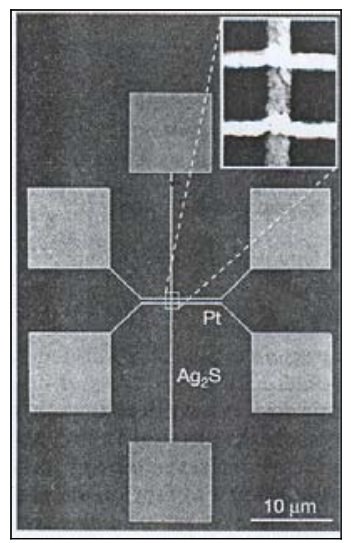

Figure 2. SEM image of the first-reported QCAS structure [1].

\section{B. Contact Pads}

Good pad contacts with the device are essential as it is the only way to control the device and determine its operating parameters. The pads were created by evaporating layers of nichrome $(20 \mathrm{~nm})$ then gold $(100 \mathrm{~nm})$ onto a 3 " diameter silicon wafer coated with silicon nitride. The substrate was then cut up into $10 \mathrm{~mm} \times 10 \mathrm{~mm}$ samples. The patterns for the contact pads was formed via optical contact lithography, as the critical feature dimensions were larger than $10 \mu \mathrm{m}$; these patterns were then transferred into the metal contact layers by a gold wet etch followed by a nichrome wet etch.

\section{Silver Wires and Sulphidation}

To create the Ag wires Electron Beam Lithography (EBL) was used to transfer 200-nm wide wire patterns into a bi-layer of poly-methylmetacrylate (PMMA) resist and then the exposed patterns were subsequently developed in an 3:1 IPA:MIBK solution. A $100-\mathrm{nm}$ thick Ag film was evaporated onto the sample, and the unwanted Ag was then lifted-off. An example of a device after this stage of processing is shown in Fig. 3, where both the $\mathrm{NiCr} / \mathrm{Au}$ contact pads and the $\mathrm{Ag}$ wires can be seen.

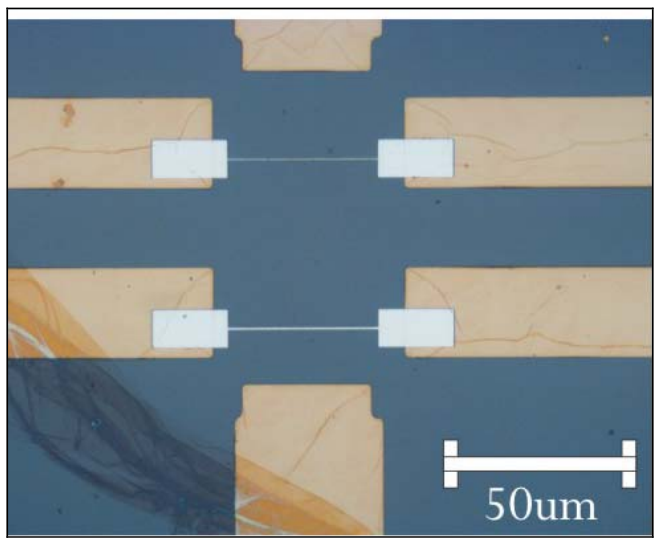

Figure 3. Optical Micrograph of a device after contact pads and Ag wires have been formed. 
The sulphidation process of Table I was used following the Ag wire lithography. To determine whether or not the wires were being sulphidised and the thickness of the sulphide layer, two different measurement techniques were used. Firstly ellipsometery on large-area silver films was used to calculate the refractive indexes of Ag control samples before and after the sulphidation process and this information was in turn used to calculate the $\mathrm{Ag}_{2} \mathrm{~S}$ thickness [7]. Secondly the resistance of the wires was measured before and after the sulphidation process and this was used to determine change in conductor cross-sectional area, from which the $\mathrm{Ag}_{2} \mathrm{~S}$ film thickness could be approximated. It was found that these two measurement methods gave comparable result, and that the $\mathrm{Ag}_{2} \mathrm{~S}$ was approximately $10 \mathrm{~nm}$ thick.

\section{Pt Crossbars and Final Structure}

The Pt crossbar was created in a manner similar to the $\mathrm{Ag}$ wires. EBL was used to transfer 200-nm width crossbar patterns into a single-layer thick PMMA instead of a thinner bilayer PMMA. A 45-nm thick film of Pt was electron-beam evaporated onto the sample and the unwanted $\mathrm{Pt}$ was subsequently lifted-off. Figure 4 shows a SEM image of one of the final devices that were created. The Pt crossbar (vertical wire), appears much wider than it actually is, which is due to the fact that there is still some hard-baked PMMA surrounding the wire (from the high-temperature of the Pt evaporation). For this device the measured width of the Pt electrode was $360 \mathrm{~nm}$ and the width of the Ag was measured to be $400 \mathrm{~nm}$, even through they were both defined as 200 -nm wide wires in the EBL pattern. This is not unusual in an EBL and lift-off process, and is due to variations in the applied exposure dose, and effects of using thicker resists; clearly some effort needs to be put into determining optical exposure doses to avoid this issue in future.

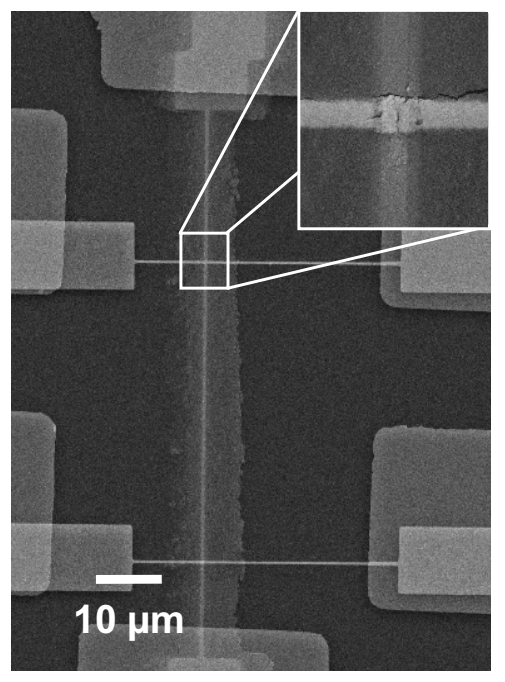

Figure 4. Scanning electron micrograph of a completed QCAS device: Sulphidised silver electrodes (horizontal) are overlaid by a platinum control electrode (vertical), with the active area shown inset. Electron beam lithography and lift off was used to form the wires.

\section{Device Testing}

Testing these devices was an involved process as the devices needed to be brought into operation by repeatedly switching them a number of times until the electrical characteristics stabilised. This was achieved using a HP 4155A parameter analyser to repeatedly apply forcing voltages and measure the current through the device. A current compliance level of $1-3 \mathrm{~mA}$ was used for these measurements, as irreversible damage was caused if the current was allowed to exceed this range.

Typical electrical characteristics are shown in Fig. 5, both during the 'soak-in' period (Fig. 5(a)) and after the device characteristics have stabilised (Fig. 5(b)). The threshold voltage for switching typically increases during the soak-in period, stabilising to a value of approximately $\pm 0.8 \mathrm{~V}$. The final switching characteristics are quite symmetrical, even though there is typically a smaller threshold voltage for turning the device OFF during the soak-in period. These devices operate with very high OFF/ON resistance ratios, up to $10^{6}$ for the device shown in Fig. 5(b). This compares very favourably to the $\mathrm{OFF} / \mathrm{ON}$ ratio of 50 for the devices of Ref. [1], indicating that our sulphidation technique is worthwhile.
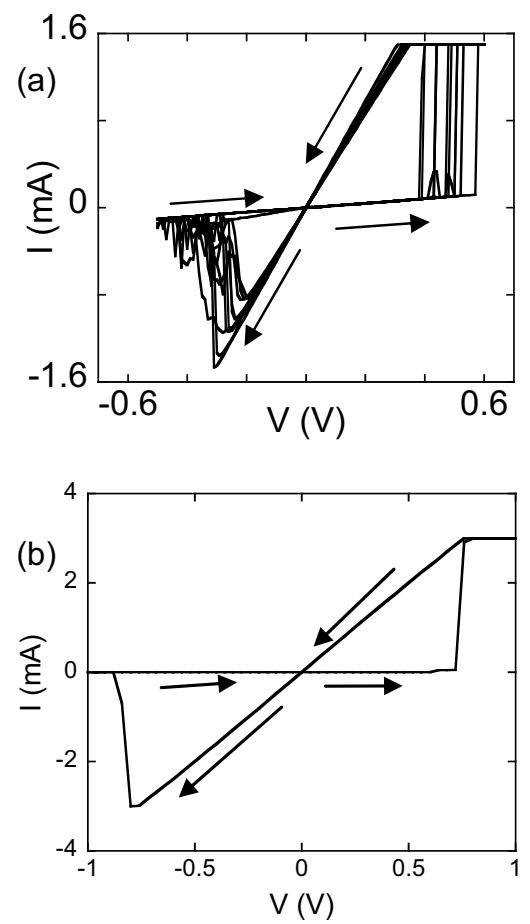

Figure 5. (a) DC current-voltage (I-V) characteristics of a QCAS device during the initial soak-in period and (b) under stable operating conditions.

The dynamic response of these devices has also been characterised, in this case using a function generator and oscilloscope for the measurements. An example of a device switching at $500 \mathrm{~Hz}$ is shown in Fig. 6, where reliable switching over 10 periods is seen. There are some instances where the switching does not occur instantaneously, even at 
these low frequencies, or where there is a 'switch bounce' type phenomenon occurring. Even though these devices operated over some thousands of cycles without changing their characteristics significantly, such transient switching effects may not be avoidable due to the fact that device operation is reliant on the mass transfer of $\mathrm{Ag}$ atoms into and out of localised nano-bridge regions. Whilst there are predictions that this has the potential to be a GHz-frequency operation [1], the reality of the nanomechanics may mean that transient effects are unavoidable. However this does not preclude the use of these devices in practical applications, and non-volatile memory is an obvious example. Whilst write times may be slow (as is the case with many current non-volatile memory technologies) the device readout time will be extremely fast, limited only by the parasitic delays in the interconnections and in the device readout circuitry.

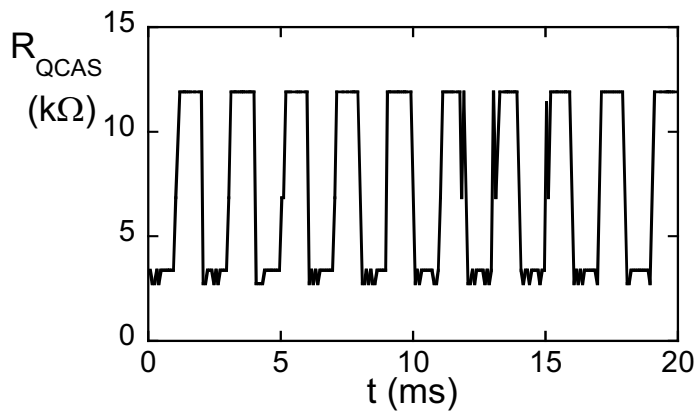

Figure 6. Switching characteristics for a QCAS device: The device is driven with a $0.75 \mathrm{~V}$ amplitude square-wave signal at $500 \mathrm{~Hz}$. Switching occurs in less than the $80 \mu$ s sampling time of the oscilloscope.

\section{CONCLUSIONS}

Quantised Conductance Atomic Switches were successfully fabricated with some interesting results. Our QCAS displayed comparable switching speeds and better resistance swing compared to those of Ref. [1], and they were fabricated with a faster and easier sulphidation process. There is plenty of research and refinement of the fabrication process to be undertaken. Particular areas that could be of interest are: improvement of processes to create devices with smoother surface electrodes and more continuous wires, reducing the size of the wire electrodes to at least that of the original devices, replication of the $1 \mathrm{~nm}$ gap between the electrodes, and most importantly adjusting the thickness of the $\mathrm{Ag}_{2} \mathrm{~S}$ film. As far as we know, using the RIE and a $\mathrm{SF}_{6}$ etch as a sulphidation process has not been reported or thoroughly researched. Considering this it would also be an important and useful to characterise this process thoroughly as it may be a useful process for other device research.

\section{ACKNOWLEDGMENTS}

Technical assistance from Helen Devereux and Gary Turner at the University of Canterbury is gratefully acknowledged for this work.

\section{REFERENCES}

[1] K. Terabe, T. Hasegawa, T. Nakayama and M. Aono, "Quantised conductance atomic switch," Nature (London), vol. 433, pp. 47-50, 2005.

[2] T. Sakamoto, H. Sunamara, H. Kawaura, T. Hasegawa, T. Nakayama and M. Aono, "Nanometre-scale switches using copper sulphide," Appl. Phys. Lett., vol 82, pp. 3032-3034, 2003.

[3] M.N. Kozicki, M. Park and M. Mitkova, "Nanoscale memory elements based on solid-state electrolytes," IEEE Trans. Nanotech., vol. 4, pp. 331-338 (2005)

[4] S. Kaeriyama T. Sakamoto, H. Sunamara, M. Mizuno, H. Kawaura, T. Hasegawa, K. Terabe, T. Nakayama and M. Aono, "A nonvolatile programmable solid-electrolyte nanometer switch,” IEEE J. Solid-State Circuits, vol. 40, pp. 168-176, 2005.

[5] G.J. Kovacs, "Sulphide Formation of Evaporated Ag Films," Surf. Sci., vol. 78, pp. L245-L249, 1978.

[6] N. Mehan and A. Mansingh, "Study of tarnished films formed on Ag by exposure to $\mathrm{H}_{2} \mathrm{~S}$ with the surface-plasmon resonance technique," Appl. Opt., vol. 39, pp. 5214-5220, October 2000.

[7] R.M.A. Azzam, N.M. Bashara, Ellipsometry and polarized light, NorthHolland, Amsterdam (1977). 